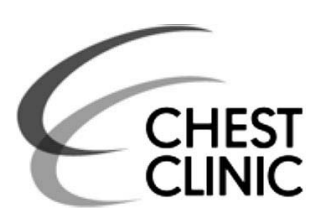

${ }^{1}$ Radiology Departament, Hospital Israelita Albert Einstein, São Paulo, São Paulo, Brazil

${ }^{2}$ Internal Medicine Departament, Hospital Israelita Albert Einstein, São Paulo, São Paulo, Brazil

Correspondence to Dr Gilberto Szarf Address: Av Albert Einstiein, 627/701, Radiology Department-

Building D, 4th Floor, São Paulo, SP 05652-000, Brazil; gszarf@yahoo.com.br

Received 19 January 2017 Revised 06 February 2017 Accepted 07 February 2017 Published Online First 28 February 2017

\title{
Herpes simplex lesion mimicking left upper lobe bronchial tumour
}

\author{
George Caldas Dantas, ${ }^{1}$ Hamilton Shoji, ${ }^{1}$ Cristiane Hoelz, ${ }^{2}$ \\ Marcelo Buarque de Gusmão Funari, ${ }^{1}$ Gilberto Szarf $^{1}$
}

A 76-year-old male patient presented with fever, cough and dyspnoea for 4 days. His history included chemotherapy for chronic lymphoid leukaemia concluded 2 years before and use of ibrutinib and hyperimmune globulin for the last year. On chest $\mathrm{X}$-ray, it was possible to identify complete atelectasis of the left upper lobe (LUL) (figure 1A, B). CT demonstrated a mass-like lesion causing obstruction of the respective lobar bronchus (figure 1C, D). The lesion showed intense focal abnormal uptake on fluorodeoxyglucose positron emission tomography/ CT (figure 2A). There were no enlarged or hypermetabolic lymph nodes. Bronchoscopy demonstrated polypoid lesions occluding the LUL bronchus (figure 2B). Bronchoalveolar lavage was negative for neoplastic cells and obtained metaplastic and mature squamous epithelial cells with cytopathic changes, compatible with viral infection.
Biopsy specimen showed bronchial mucosa fragments with papillary projections lined by stratified squamous and columnar respiratory epithelium, without atypical cells. Five days later, a new bronchoscopic biopsy was performed, which demonstrated extensive mucosal thickening, with debris; epithelial cells demonstrated nuclear inclusion bodies, consistent with herpes simplex virus (HSV) infection (figure 2C). Molecular testing using PCR identified HSV type 1 and 2 in tissue specimen. The treatment included bronchoscopic argon plasma coagulation and oral acyclovir for 6 weeks. CT after treatment revealed regression of the lesion with re-expansion of the LUL (figure 2D).

Pulmonary HSV infection is rare and usually occurs in immunocompromised patients, although tracheobronchitis and bronchopneumonia have also been reported in immunocompetent patients. ${ }^{1-3}$
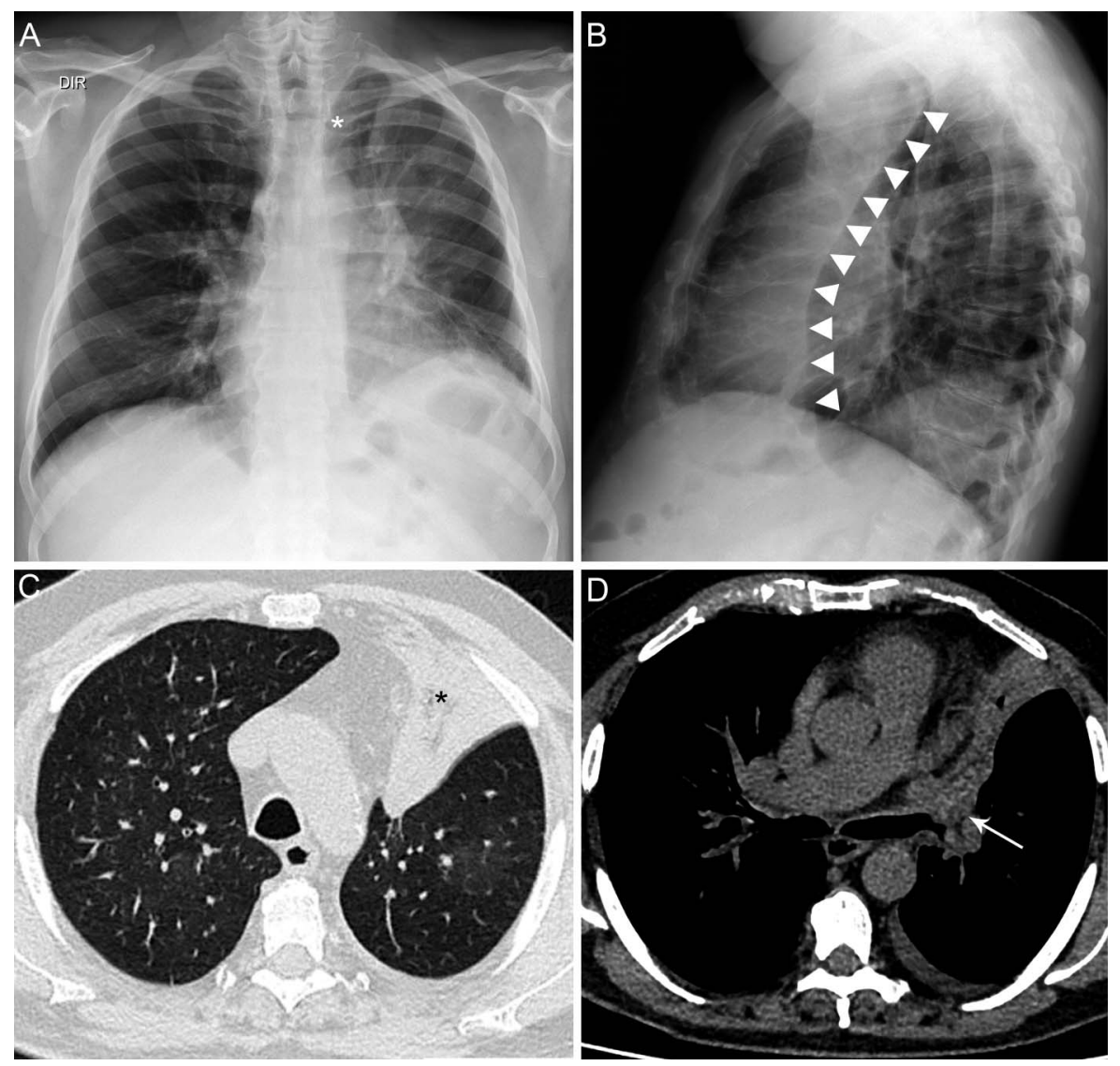

To cite: Dantas GC, Shoji H, Hoelz C, et al. Thorax 2018;73:94-95.

Figure 1 Posteroanterior (A) and lateral (B) chest radiography showing left upper lobe (LUL) collapse (arrowheads). There is a para-aortic crescent of hyperlucency (white *) on the posteroanterior view (Luftsichel sign). Chest CT confirming the LUL collapse (black ${ }^{*}, \mathrm{C}$ ) and obliteration of the corresponding lobar bronchus (arrow, D). 
Figure 2 (A) Positron emission tomography-CT demonstrating intense focal fluorodeoxyglucose metabolism (arrow) within the lesion occluding the left upper lobe bronchus. (B) Corresponding polipoid lesion on bronchoscopic view. (C) H\&E stain biopsy specimen showing necrotic debris and epithelial cells with nuclear inclusion bodies (black arrows), consistent with herpes simplex virus infection. (D) Follow-up CT after bronchoscopic argon plasma coagulation and clinical treatment, with desobstruction of the left upper lobe bronchus and lung reexpansion; some residual irregularities (arrowheads) are visible.
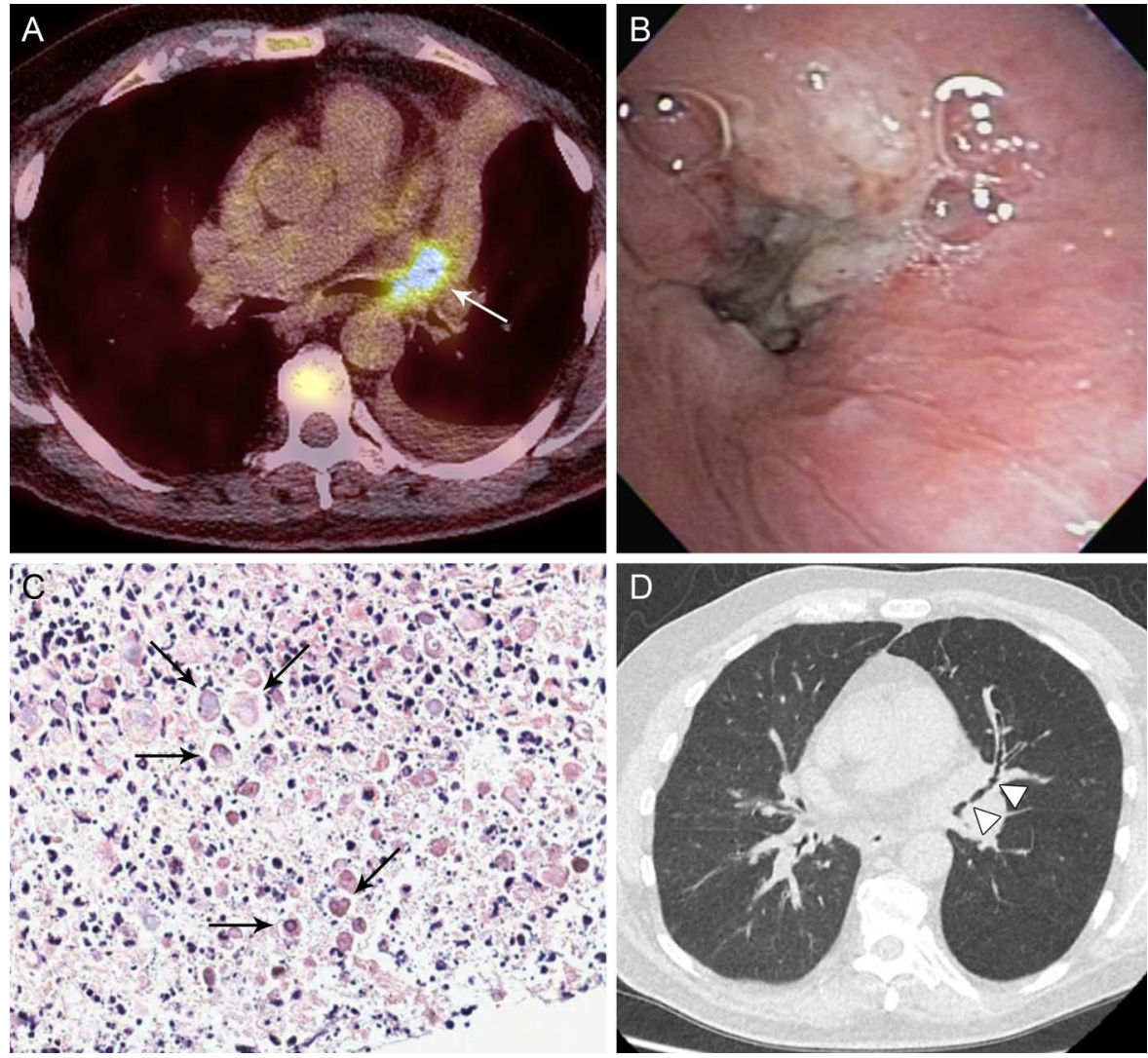

The pathophysiology is not well established, but it has been postulated that the virus can reach the lower respiratory tract by aspiration or by contiguous propagation after reactivation in the oropharynx, and there may be dissemination after orotracheal intubation. The presence of macroscopic bronchial lesions is possibly related to local microtraumas and/or to previous pulmonary infections that determined squamous metaplasia. ${ }^{3}$

Diagnosis is based on nuclear inclusion identified on cytological analysis in samples obtained from bronchoscopic examination and on the detection of HSV by virus culture or, more commonly, quantitatively by nucleic acid amplification techniques. ${ }^{2} 3$

Although uncommon, infection of the lower respiratory tract by herpes virus is possible, even simulating a tumour. An accurate diagnosis of this entity should be performed, because it totally changes the perspective of patient treatment and prognosis.

Acknowledgements The authors would like to thank Andrea Puchnick for photographic and editing assistance and Christina Shiang, lunis Suzuki and Altair Costa Junior for the contribution in the case.
Collaborators Christina Shiang, lunis Suzuki, Altair Costa Junior.

Contributors GCD: conception or design of the work, data collection, data analysis and interpretation. HS: conception or design of the work, data collection, data analysis and interpretation. $\mathrm{CH}$ : data collection, data analysis and interpretation. MBGF: conception or design of the work, data collection, data analysis and interpretation. GS: conception or design of the work, data analysis and interpretation, drafting the article, critical revision of the article. Final approval of the version to be published.

Competing interests None declared.

Patient consent Obtained.

Provenance and peer review Not commissioned; externally peer reviewed.

\section{REFERENCES}

1 Armbruster C, Drlicek M. Herpes simplex virus type II infection as an exophytic endobronchial tumor. Wien Klin Wochenschr 1995;107:344-6.

2 Upadya A, Tilluckdharry L, Nagy CD, et al. Endobronchial pseudo-tumour caused by herpes simplex. Eur Respir J 2005;25:1117-20.

3 Katsenos S, Sampaziotis D, Archondakis S. Tracheal pseudo-tumor caused by herpes simplex virus. Multidiscip Respir Med 2013;8:42. 